\title{
Judgment aggregators and Boolean algebra homomorphisms
}

\author{
Frederik Herzberg \\ Copyright notice: The final publication, in the Journal of Mathematical \\ ECONOMICS 46 (2010), no. 1, pp. 132-140, is available at sciencedirect.com, \\ doi:10.1016/j.jmateco.2009.06.002
}

\begin{abstract}
The theory of Boolean algebras can be fruitfully applied to judgment aggregation: Assuming universality, systematicity and a sufficiently rich agenda, there is a correspondence between (i) nontrivial deductively closed judgment aggregators and (ii) Boolean algebra homomorphisms defined on the power-set algebra of the electorate. Furthermore, there is a correspondence between (i) consistent complete judgment aggregators and (ii) 2-valued Boolean algebra homomorphisms defined on the power-set algebra of the electorate.

Since the shell of such a homomorphism equals the set of winning coalitions and since (ultra)filters are shells of (2-valued) Boolean algebra homomorphisms, we suggest an explanation for the effectiveness of the (ultra)filter method in social choice theory.

From the (ultra)filter property of the set of winning coalitions, one obtains two general impossibility theorems for judgment aggregation on finite electorates, even without assuming the Pareto principle.
\end{abstract}

2000 Mathematics Subject Classification. 91B14; 03G05.

Journal of Economic Literature classification. D71.

Key words and phrases. Judgment aggregation; systematicity; impossibility theorems; filter; ultrafilter; Boolean algebra homomorphism.

Institut für Mathematische Wirtschaftsforschung, Universität Bielefeld, Universitätsstraße

D-33615 Bielefeld, Germany. Email address: fherzberg@uni-bielefeld.de.

Department of Mathematics, University of California, Berkeley, CA 94720-3840, United States of America. Email address: herzberg@math.berkeley.edu.

I would like to thank Professor Franz Dietrich, Daniel Eckert and Bernard Monjardet for various helpful comments. 


\section{Introduction}

The purpose of the present paper is three-fold: (i) To show that universal systematic judgment aggregation can be studied by means of Boolean algebras. (ii) To show that the concept of an (ultra)filter arises naturally when describing systematic judgment aggregators through Boolean algebras, and thus to explain the effectiveness of the (ultra)filter methodology in abstract aggregation theory. (iii) To give concise algebraic proofs of dictatorial and oligarchic impossibility theorems in judgment aggregation. The thrust of this paper is the second aspect which suggests a new perspective on the (ultra)filter method.

The use of filters and ultrafilters has been firmly established in the theory of preference aggregation. Fishburn [8] was the first to apply the ultrafilter concept to prove a possibility theorem for preference aggregation on infinite electorates, and Kirman and Sondermann $1 \mathbf{1 3}$ employed the ultrafilter concept to prove that Arrow's rationality axioms ${ }^{1}$ imply dictatorship for finite electorates. Slightly later, Hansson [11] and Brown [3] realized that the concept of a filter can be used to characterize oligarchies: ${ }^{2}$ This so-called (ultra)filter methodology can be summarized as follows: In order to prove impossibility theorems for finite electorates, one shows, using rationality axioms on the aggregation function, that the set of all decisive coalitions must be an ultrafilter (filter, respectively) on the power-set of the electorate. If the electorate is finite, one can then deduce that this set of coalitions must be the set of all coalitions containing one and the same element (one and the same subset, repectively), viz. the dictator (set of oligarchs, respectively).

During the past two years, the (ultra)filter method has also been applied in the theory of judgment aggregation (i.e. aggregation of logical propositions): Eckert and Klamler [7] employ ultrafilters to prove a simple dictatorial impossibility theorem for judgment aggregation due to Nehring and Puppe [17], and Dietrich and Mongin [5] prove more general impossibility results for judgment aggregation, of both oligarchic and dictatorial kind, by means of (ultra)filters.

The success of the (ultra)filter method in social choice theory has often been simply attributed to the fact that filters and ultrafilters possess, apparently by some mathematical coincidence, exactly those set-theoretic closure properties that are also desirable in the investigation of economic aggregation problems. A notable exception is an article by Lauwers and Van Liedekerke [14] which shows that there is a one-to-one correspondence between ultraproduct: $\mathrm{s}^{3}$ of preference relations on a given set of alternatives and preference aggregation functions.

\footnotetext{
${ }^{1}$ By "Arrovian rationality axioms" we mean the following four conditions: at least three alternatives; universality; unanimity preservation (Pareto principle); independence of irrelevant alternatives.

${ }^{2}$ Monjardet [16] has argued that Guilbaud's analysis of collective decision making [9] (English translation in [10]), which appeared around the time of Arrow's classic Social Choice and Individual Values, implicitly uses the notion of an ultrafilter.

${ }^{3} \mathrm{An}$ ultraproduct is a model-theoretic construction, obtained from a - finite or infinite - sequence of structures of the same type, based on an ultrafilter on the index set of the sequence.
} 
However, one can give a more fundamental explanation for the link between abstract aggregation theory and (ultra)filters: On the one hand, the concept of a Boolean algebra is a natural mathematical notion for formalizing abstract aggregation theory as it provides a unified framework to capture both the algebraic structure of the electorate and the algebraic structure of the set of truth values. On the other hand, (ultra)filters occur naturally in the context of Boolean algebras because homomorphisms of Boolean algebras are typically classified via the pre-image of the 1-element of the image algebra, called shell, and shells of (2-valued) Boolean algebra homomorphisms are nothing else than (ultra)filters.

In light of this, it is reasonable to ask whether judgment aggregators can, at least under some rationality assumptions, be conceived of as Boolean algebra homomorphisms and, if so, whether such a representation allows for an economic interpretation of the shell of a homomorphism induced by a judgment aggregator.

For sufficiently rich agendas, the answer to both questions turns out to be affirmative.

Assuming universality, systematicity, and an agenda richness condition, we show through Theorem 3 ,

- Consistent and complete judgment aggregators correspond to 2valued Boolean algebra homomorphisms defined on the power-set algebra of the electorate.

- The shell of any such homomorphism is just the set of all winning coalitions (i.e. those coalitions which win the collective outcome for some proposition and some profile).

- Hence the set of all winning coalitions is an ultrafilter if the judgment aggregator is consistent, complete, universal and systematic.

Theorem 6 generalizes this result. Again assuming universality, systematicity, and an agenda richness condition:

- Non-trivial deductively closed judgment aggregators correspond to Boolean algebra homomorphisms defined on the power-set algebra of the electorate.

- The shell of every such homomorphism equals the set of all winning coalitions.

- Hence the set of all winning coalitions is a filter if the judgment aggregator is non-trivial, deductively closed, universal and systematic.

Under the additional assumption of a finite electorate, we obtain a dictatorial impossibility theorem for certain complete systematic judgment aggregators and an oligarchic impossibility theorem for certain deductively closed systematic aggregators. These impossibility theorems are among the most general versions of Arrow's theorem to this day, and are slight variations of results by Dietrich and List [4] and Dietrich and Mongin [5]. The algebraic 
methodology of this paper allows us to give remarkably short proofs of these results 4

The paper presents the framework in Section 2 and Section 3 . Thereafter, one section each is devoted to the axioms on judgment aggregators, the results, the proofs, and a conclusion. Technical footnotes make this article self-contained.

\section{Judgment sets}

Let $X$ be a set of sentences in propositional logic, called the agenda. We assume that $X$ is the union of proposition-negation pairs (i.e. there exists a non-empty set $X^{\prime}$ of sentences such that $\left.X=\bigcup_{p \in X^{\prime}}\{p, \neg p\}\right)$. For every $p \in X$ we denote by $\sim p$ an element $q$ of $X$ such that either $q=\neg p$ or $p=\neg q$.

Subsets of $X$ will be called judgment sets, and we denote the power-set of $X$ by $\mathcal{P}(X)$.

Let $\Sigma$ be a consistent set of sentences in propositional logic $5^{5}$

For every judgment set $Y$ :

(1) $Y$ is $\Sigma$-consistent if and only if $\Sigma \cup Y \forall \forall(p \wedge \neg p)$ for any sentence $p$.

(2) $Y$ is $\Sigma$-deductively closed (in $X$ ) if and only if for all $p \in X$, if $\Sigma \cup Y \vdash p$, then $p \in Y$.

(3) $Y$ is complete (in $X$ ) if and only if for all $p \in X, p \notin Y$ implies $\sim p \in Y$.

(4) $Y$ is algebraically consistent if and only if for all $p \in X, \sim p \in Y$ implies $p \notin Y$.

Clearly, every $\Sigma$-consistent set is algebraically consistent. The converse is true if the elements of $\Sigma \cup X^{\prime}$ consist of pairwise different propositional variables, hence the name algebraic, as opposed to logical, consistency.

We assume that for every $p \in X,\{p\}$ is $\Sigma$-consistent. Let us introduce the following abbreviations:

(1) $D$ denotes the set of all $\Sigma$-consistent and complete subsets of $X$.

(2) $D^{*}$ denotes the set of all $\Sigma$-consistent and $\Sigma$-deductively closed subsets of $X$.

\footnotetext{
${ }^{4}$ After dissemination of the present paper, Bernard Monjardet informed the author that a similar Boolean view of aggregation can also be found in some of the literature on preference aggregation (cf. Aleskerov [1] or Kim and Roush [12]).

${ }^{5}$ Many authors of the judgment-aggregation literature formulate their results with respect to a general monotonic logic $L$ instead of propositional logic. In order to translate our framework into theirs, we have to assume that $X$ is a set of $L$-formulae, where $L$ is a language whose symbols include the connectives $\neg$ and $\wedge$ (other truth-functional connectives are defined as usual in terms of $\neg$ and $\wedge$ ). We need to require, in addition, that a formal provability relation $\vdash$ is defined for sets of $L$-formulae, and assume that the system of consistent subsets induced by $\vdash$ has the following properties:

(1) $\{p, \neg p\}$ is inconsistent for every $L$-formula $p$;

(2) subsets of consistent sets are consistent (monotonicity);

(3) $\varnothing$ is consistent, and every consistent set has a consistent superset containing an element of each pair-set $\{p, \neg p\}$;

(4) for all $L$-formulae $p, q,\{p, q\} \vdash p \wedge q$ as well as both $p \wedge q \vdash p$ and $p \wedge q \vdash q$.

Note that in this framework, $\Sigma$ can simply be added to the axioms of the deductive system given by $\vdash$. Hence, if one adopts this general framework, $\Sigma$ is redundant and may be deleted - or replaced by $\varnothing$ - wherever it occurs in the following. (I owe the formulation of this footnote to Professor Franz Dietrich.)
} 
(3) $D^{\prime}$ denotes the set of all $\Sigma$-deductively closed subsets of $X$.

(4) $D^{a c}$ denotes the set of all algebraically consistent and complete subsets of $X$.

(5) $D^{a}$ denotes the set of all algebraically consistent subsets of $X$.

A subset $Y \subseteq X$ is $\in D^{a c}$ if and only if for all $p \in X$,

$$
p \notin Y \Leftrightarrow \sim p \in Y \text {. }
$$

Clearly $D \subseteq D^{*} \subseteq D^{\prime}$, and $D \subseteq D^{a c} \subseteq D^{a}$.

We shall often suppress the specification " $\Sigma$ "; we shall write 'consistent' instead of ' $\Sigma$-consistent' and 'deductively closed' instead of ' $\Sigma$-deductively closed'

\section{Judgment aggregators and coalitions}

Consider a non-empty (finite or infinite) set $N$, called the electorate (committee/population) set. The elements of $N$ will be referred to as individuals, and subsets of $N$ will be called coalitions. We denote the powerset of $N$ by $\mathcal{P}(N)$.

Consider some map $f: \mathfrak{D}_{f} \rightarrow \mathcal{P}(X)$ with $\varnothing \neq \mathfrak{D}_{f} \subseteq\left(D^{\prime}\right)^{N}$, called judgment aggregator. Elements of the domain $\mathfrak{D}_{f}$ of $f$ will be referred to as profiles, the components of every profile will be called individual judgment sets, whereas the elements of the range of $f$ will be called collective judgment sets.

$f$ is called complete (or consistent, or deductively closed, or algebraically consistent, respectively) if its range only consists of complete (or consistent, or deductively closed, or algebraically consistent, respectively) judgment sets ${ }^{6}$

There are two kinds of impossibility theorems: dictatorial and oligarchic ones. $f$ will be called dictatorial if and only if there exists some $i_{f} \in N$ such that $f(\underline{A})=A_{i_{f}}$ for all $\underline{A} \in \mathfrak{D}_{f} . f$ will be called oligarchic if and only if there exists some non-empty $M_{f} \subseteq N$ such that $f(\underline{A})=\bigcap_{i \in M_{f}} A_{i}$ for all $\underline{A} \in \mathfrak{D}_{f}$.

Next, we introduce some terminology and notation for the description of coalitions. For all $p \in X$ and $\underline{A}=\left\langle A_{i}\right\rangle_{i \in N} \in \mathfrak{D}_{f}$, the coalition

$$
\underline{A}(p):=\left\{i \in N: p \in A_{i}\right\}
$$

is called the coalition supporting $p$ given $\underline{A}$. We say that $\underline{A}(p)$ is winning for $p$ given $\underline{A}$ under $f$ if and only if $p \in f(\underline{A})$.

We collect all winning coalition $\$^{7}$ in the set

$$
\mathcal{F}_{f}:=\left\{\underline{A}(p): \underline{A} \in \mathfrak{D}_{f}, \quad p \in f(\underline{A})\right\},
$$

\footnotetext{
${ }^{6}$ In the first part of Theorem 6, we shall only assume that $f$ is deductively closed, hence we do not have to assume completeness or consistency at the collective level. A similar framework has been suggested by Dietrich and List [4]. The consistency of the collective judgment sets will follow from other properties of the judgment aggregators under consideration.

7 The set of winning coalitions for $p$ is the same for each profile if and only if $f$ is independent, i.e. for every $p \in X$ and $\underline{A}, \underline{A}^{\prime} \in \mathfrak{D}_{f}$,

$$
\underline{A}(p)=\underline{A}^{\prime}(p) \Rightarrow\left(p \in f(\underline{A}) \Leftrightarrow p \in f\left(\underline{A}^{\prime}\right)\right) .
$$
}


and we say that two coalitions $C, C^{\prime} \subseteq N$ are said to share the same part of a winning coalition (denoted $C \sim_{f} C^{\prime}$ ) if and only if there exists some $U \in \mathcal{F}_{f}$ such that

$$
C \cap U=C^{\prime} \cap U .
$$

These two concepts - winning coalition and sharing the same part of a winning coalition - will be useful in the algebraic description of systematic judgment aggregators.

Finally, we adopt the following convention as a notational device: For all $\underline{A} \in \mathfrak{D}_{f}$, we regard $f(\underline{A})$ as a function $f(\underline{A}): X \rightarrow\{0,1\}$, defined via

$$
f(\underline{A})(p)= \begin{cases}1, & p \in f(\underline{A}) \\ 0, & p \notin f(\underline{A})\end{cases}
$$

\section{Axioms}

Consider the following axioms, which are inspired by Arrow's rationality axioms:

- A1. Finite electorate. $N$ is finite.

- A2. Agenda richness. There are propositions $p, q \in X$ such that each of the propositions $p \wedge q, \neg p \wedge q, p \wedge \neg q$ is $\Sigma$-consistent and $\in X$.

- A3. Universality. $\mathfrak{D}_{f} \supseteq D^{N}$.

- A4. Non-triviality. $f$ is neither constantly $=\varnothing$ nor constantly $=X$.

- A5. Systematicity. For all $p, q \in X$ and $\underline{A}, \underline{A}^{\prime} \in \mathfrak{D}_{f}$ : If $\underline{A}(p)=$ $\underline{A}^{\prime}(q)$, then

$$
p \in f(\underline{A}) \Leftrightarrow q \in f\left(\underline{A}^{\prime}\right) .
$$

The axiom of non-triviality, which to the knowledge of the author is new in the judgment-aggregation literature, is satisfied in two important special cases:

REMARK 1. (1) Suppose $f$ satisfies (A3) as well as the axiom of strict unanimity preservation, i.e. for all $p \in X$ and $\underline{A} \in \mathfrak{D}_{f}$, if $\underline{A}(p)=N$ then $p \in f(\underline{A})$ and if $\underline{A}(p)=\varnothing$ then $p \notin f(\underline{A})$. Then $f$ satisfies (A4).

(2) Since $\varnothing$ is not a complete subset and $X$ not a consistent subset of $X$, one has $\varnothing \notin D$ and $X \notin D$. Therefore, (A4) is satisfied if $f$ is both complete and consistent.

Proof. (1) Since $X$ is the union of proposition-negation pair-sets, it must contain some consistent proposition $p$ and some proposition $q$ which is not universally valid. Hence there must be some $\underline{A} \in D^{N}$ with $\underline{A}(p)=N$ and some $\underline{A}^{\prime} \in D^{N}$ with $\underline{A}^{\prime}(q)=\varnothing$. If $f$ satisfies both (A3) and strict unanimity preservation, then $p \in f(\underline{A})$ and $q \notin f\left(\underline{A^{\prime}}\right)$.

(2) $\varnothing$ is incomplete, and $X$ is inconsistent (being the union of proposition-negation pair-sets). So, if $f$ is both complete and consistent, then $f(\underline{A}) \neq \varnothing$ and $f(\underline{A}) \neq X$ for all $\underline{A} \in \mathfrak{D}_{f}$. 
The axiom of systematicity clearly implies the axiom of independence $8^{8}$ Actually, systematicity is even equivalent to independence if the agenda satisfies an additional condition known as total blockedness (cf. e.g. Eckert and Klamler [7]). Intuitively, an agenda is totally blocked if "any proposition in the agenda can be reached from any other proposition in it via a sequence of conditional entailments" (List and Puppe [15]), in other words, if one can deduce "any position on any issue from any position on any issue, via a chain of deductions" (Dokow and Holzman [6]).

The agenda richness axiom is inspired by the ultrafilter proof of Lauwers and Van Liedekerke [14.

\section{Results}

Recall that $\langle\mathcal{P}(N), \cap, \cup, \complement, \varnothing, N\rangle$ (wherein $\complement B:=N \backslash B$ for all $B \subseteq N$ ) and $\left\langle\{0,1\}, \wedge, \vee,^{*}, 0,1\right\rangle$ (wherein $0^{*}=1,1^{*}=0$ ), are Boolean algebras $9^{9}$ We write 2 for $\{0,1\}$. We will show that non-trivial universal systematic judgment aggregators are derived from Boolean algebra homomorphisms ${ }^{10}$ with domain $\mathcal{P}(N)$ and vice versa. The shell ${ }^{11}$ of these homomorphisms will be just the set of winning coalitions.

LEMma 2. If $f$ satisfies (A2), (A3) and (A5), then the map

$$
\pi: \mathcal{P}(N) \rightarrow 2, \quad \underline{A}(p) \mapsto f(\underline{A})(p)
$$

is well-defined. If, in addition, $f$ is deductively closed, then $\pi^{-1}\{1\}$ equals $\mathcal{F}_{f}$ and is both $\supseteq$-closed and $\cap$-closed ${ }^{12}$

\footnotetext{
${ }^{8}$ See Footnote 7 on page 5

${ }^{9}\left\langle B, \wedge, \vee,{ }^{*}, 0_{B}, 1_{B}\right\rangle$ is called a Boolean algebra if and only if $\wedge$ and $\vee$ are associative and commutative operations on a non-empty set $B$ satisfying both

$$
\forall x, y \in B \quad(x \vee y) \wedge y=(x \wedge y) \vee y=y
$$

and the distributivity axiom

$$
\forall x, y, z \in B \quad(x \vee y) \wedge z=(x \wedge z) \vee(y \wedge z)
$$

and for all $x \in B$, the element $x^{*} \in B$ satisfies

$$
x \wedge x^{*}=0_{B}, \quad x \vee x^{*}=1_{B} .
$$

Every Boolean algebra can be endowed with an antisymmetric, reflexive and transitive ordering by defining

$$
x \leq y: \Leftrightarrow x \wedge y=x
$$

for all $x, y \in B$. For a primer of Boolean algebras and their use in logic, cf. e.g. Bell and Slomson [2, Chapter 1].

${ }^{10} \mathrm{~A}$ Boolean algebra homomorphism, for short: homorphism, is a map $\rho: B_{1} \rightarrow$ $B_{2}$ between two Boolean algebras $\left\langle B_{1}, \wedge_{1}, \vee_{1},{ }^{*}, 0_{1}, 1_{1}\right\rangle$ and $\left\langle B_{2}, \wedge_{2}, \vee_{2},{ }^{-}, 0_{2}, 1_{2}\right\rangle$ which preserves all three Boolean operations, i.e.

$$
\rho\left(x \wedge_{1} y\right)=\rho(x) \wedge_{2} \rho(y), \quad \rho\left(x \vee_{1} y\right)=\rho(x) \vee_{2} \rho(y), \quad \rho\left(x^{*}\right)=\rho(x)^{-}
$$

for all $x, y \in B_{1}$.

${ }^{11}$ The shell of a Boolean algebra homomorphism $\rho: B_{1} \rightarrow B_{2}$ is defined as $\rho^{-1}\left\{1_{2}\right\}$.

${ }^{12}$ In other words, if $C, C^{\prime} \in \mathcal{F}_{f}$ and $C^{\prime \prime} \supseteq C$, then both $C \cap C^{\prime} \in \mathcal{F}_{f}$ and $C^{\prime \prime} \in \mathcal{F}_{f}$. A non-empty subset $\mathcal{G}$ of $\mathcal{P}(N)$ that is both $\supseteq$-closed and $\cap$-closed is sometimes already called filter. However, we use the word filter in the sense of proper filter and require in addition that $\mathcal{G} \neq \mathcal{P}(N)$.
} 
Note that $\pi$ does not have to be a lattice homomorphism ${ }^{13}$ (let alone a Boolean algebra homomorphism) in general: $\pi$ need not preserve joins, since the union of two coalitions $C, C^{\prime}$ which are not winning (i.e. $C, C^{\prime} \in \pi^{-1}\{0\}$ ) can nevertheless be a winning coalition (i.e. $C \cup C^{\prime} \in \pi^{-1}\{1\}$ ) ${ }^{14}$

Our first result is that complete $\Sigma$-consistent non-trivial systematic judgment aggregators correspond to 2-valued Boolean algebra homomorphisms, and that every such homomorphism yields a complete algebraically consistent systematic judgment aggregator.

TheOrem 3. (1) If $f$ satisfies (A2), (A3) and (A5) and is both consistent and complete, then $f$ also satisfies (A4) and $\pi$ is a homomorphism with shell $\mathcal{F}_{f}$.

(2) Conversely, if $\rho: \mathcal{P}(N) \rightarrow 2$ is a homomorphism and (A2) is satisfied, then the judgment aggregator

$$
f: D^{N} \rightarrow \mathcal{P}(X), \quad \underline{A} \mapsto\{p \in X: \rho(\underline{A}(p))=1\}
$$

satisfies (A2-A5) and is both algebraically consistent and complete.

Corollary 4. If $f$ satisfies (A2), (A3) and (A5) and is both consistent and complete, then $\mathcal{F}_{f}$ is an ultrafilter ${ }^{15}$ If, in addition, (A1) holds, then $f$ is dictatorial.

In the following we mean by a congruence relation an equivalence relation which respects the Boolean operations ${ }^{16}$ Recall that two coalitions $C, C^{\prime}$ stand in relation $\sim_{f}$ to each other if and only if they share the same part of some winning coalition.

LEMma 5. If $f$ satisfies axioms (A2-A5) and is deductively closed, then $\sim_{f}$ is a congruence relation on the Boolean algebra $\mathcal{P}(N)$ and the Boolean operations on $\mathcal{P}(N)$ induce a Boolean algebra structure on $\mathcal{P}(N) / \sim_{f}$.

Theorem 3 can be generalized as follows:

THEOREM 6. (1) If $f$ satisfies (A2-A5) and is deductively closed, then the canonical surjection $\sigma: \mathcal{P}(N) \rightarrow \mathcal{P}(N) / \sim_{f}$ is a homomorphism with shell $\mathcal{F}_{f}$.

(2) Conversely, if $\tau: \mathcal{P}(N) \rightarrow B$ is a homomorphism for some Boolean algebra $B$ and (A2) is satisfied, then the judgment aggregator

$$
f: D^{N} \rightarrow \mathcal{P}(X), \quad \underline{A} \mapsto\left\{p \in X: \tau(\underline{A}(p))=1_{B}\right\},
$$

satisfies (A2-A5) and is algebraically consistent.

\footnotetext{
${ }^{13}$ A lattice homomorphism is a map between two lattices, e.g. Boolean algebras, which preserves meets and joins.

${ }^{14}$ Consider, for example, an oligarchic $f$ and let $C$ be a proper subset of the set $M_{f}$ of oligarchs and let $C^{\prime}=M_{f} \backslash C$. Then, $C, C^{\prime} \in \pi^{-1}\{0\}$ albeit $C \cup C^{\prime}=M_{f} \in \pi^{-1}\{1\}$.

${ }^{15}$ A non-empty set $\mathcal{G} \subseteq \mathcal{P}(N)$ is called a filter on $N$ if and only if $\mathcal{G} \neq \mathcal{P}(N)$ and $\mathcal{G}$ is both $\supseteq$-closed and $\cap$-closed. A filter is called an ultrafilter if and only if for all $C \subseteq N$, either $C \in \mathcal{G}$ or $N \backslash C \in \mathcal{G}$.

${ }^{16}$ More formally, a binary relation $\sim_{C}$ on a Boolean algebra is a congruence relation if and only if $\sim_{C}$ is reflexive, symmetric and transitive, and for all $x, y, x^{\prime}, y^{\prime}$ such that both $x \sim_{C} x^{\prime}$ and $y \sim_{C} y^{\prime}$, one has

$$
x \wedge y \sim_{C} x^{\prime} \wedge y^{\prime}, \quad x \vee y \sim_{C} x^{\prime} \vee y^{\prime}, \quad x^{*} \sim_{C}\left(x^{\prime}\right)^{*} .
$$
}


COROllary 7. If $f$ satisfies axioms (A2-A5) and is deductively closed, then $\mathcal{F}_{f}$ is a filter. If, in addition, (A1) holds, then $f$ is oligarchic.

\section{Concise algebraic proofs}

REMARK 8. Let $\kappa$ be finite or infinite. Let $N=\bigcup_{j \in \kappa} C_{j}$ be a disjoint decomposition of $N$ and let $\left\langle Y_{j}\right\rangle_{j \in \kappa}$ be a family of $\Sigma$-consistent subsets of $X$. Then, each $Y_{j}$ can be extended to a $\Sigma$-consistent and complete subset $Z_{j}$ of $X$. Thus, there exists some profile $\underline{A} \in D^{N}$ such that $A_{i}=Z_{j} \supseteq Y_{j}$ for every $i \in C_{j}$ and $j \in \kappa$.

Remark 9. If (A2) is satisfied, then $\left\{\underline{A}(p): \underline{A} \in D^{N}, \quad p \in X\right\}=$ $\mathcal{P}(N)$.

Proof. Due to (A2), $X$ contains a sentence $p$ such that both $\{p\}$ and $\{\neg p\}$ are $\Sigma$-consistent ${ }^{17}$ Consider now an arbitrary coalition $C$. Remark 8 provides a profile $\underline{A} \in D^{N}$ such that for all $i \in N$, if $i \in C$ then $p \in A_{i}$ and if $i \in N \backslash C$ then $\neg p \in A_{i}$, hence $p \notin A_{i}$ whenever $i \in N \backslash C$ since $A_{i}$ is consistent. Therefore,

$$
p \in A_{i} \Leftrightarrow i \in C
$$

for every $i \in N$. Hence $\underline{A}(p)=C$. So, every coalition $C$ is of the form $\underline{A}(p)$ for some $\underline{A} \in D^{N}$ and $p \in X$.

Proof of Lemma 2, Suppose $f$ satisfies (A2), (A3) and (A5). By (A5), $\pi$ is well-defined on $\mathfrak{D}_{\pi}:=\left\{\underline{A}(p): \underline{A} \in \mathfrak{D}_{f}, \quad p \in X\right\}$. Since $\mathfrak{D}_{f} \supseteq$ $D^{N}$ by (A3), we actually have $\mathfrak{D}_{\pi} \supseteq\left\{\underline{A}(p): \underline{A} \in D^{N}, \quad p \in X\right\}$, hence $\mathfrak{D}_{\pi}=\mathcal{P}(N)$ by Remark 9 .

Since

$$
f(\underline{A})(p)=1 \Leftrightarrow p \in f(\underline{A})
$$

for all $p \in X$ and $\underline{A} \in \mathfrak{D}_{f}$, it is clear that $\pi^{-1}\{1\}=\mathcal{F}_{f}$. Next, we shall prove that $\pi^{-1}\{1\}$ is closed under supersets and intersections. For the following, let $p, q$ denote the two sentences whose existence was postulated in (A2).

$\pi^{-1}\{1\}$ is $\supseteq$-closed. Let $C^{\prime} \in \pi^{-1}\{1\}$ and $C \supseteq C^{\prime}$. By (A2) and Remark 8 there exists a profile $\underline{A} \in D^{N}$ such that

$\forall i \in C \backslash C^{\prime} \quad p \wedge \neg q \in A_{i}, \quad \forall i \in N \backslash C \quad \neg p \wedge q \in A_{i}, \quad \forall i \in C^{\prime} \quad p \wedge q \in A_{i}$.

Then $\underline{A}(p \wedge q)=C^{\prime} \in \pi^{-1}\{1\}$, whence $p \wedge q \in f(\underline{A})$ because $\pi$ is welldefined. However, $f(\underline{A})$ is deductively closed, therefore $p \in f(\underline{A})$, hence $\pi^{-1}\{1\} \ni \underline{A}(p)=\left(C \backslash C^{\prime}\right) \cup C^{\prime}=C$.

$\pi^{-1}\{1\}$ is $\cap$-closed. Let $C^{\prime}, C^{\prime \prime} \in \pi^{-1}\{1\}$. By (A2) and Remark 8 there exists a profile $\underline{A}^{\prime} \in D^{N}$ such that

$\forall i \in C^{\prime \prime} \backslash C^{\prime} \quad p \wedge \neg q \in A_{i}^{\prime}, \quad \forall i \in N \backslash C^{\prime \prime} \quad \neg p \wedge q \in A_{i}^{\prime}, \quad \forall i \in C^{\prime} \cap C^{\prime \prime} \quad p \wedge q \in A_{i}^{\prime}$. Then $\underline{A}^{\prime}(p)=\left(C^{\prime} \cap C^{\prime \prime}\right) \cup\left(C^{\prime \prime} \backslash C^{\prime}\right)=C^{\prime \prime} \in \pi^{-1}\{1\}$, so $p \in f\left(\underline{A}^{\prime}\right)$ since $\pi$ is well-defined. On the other hand, $\underline{A}^{\prime}(q)=\left(C^{\prime} \cap C^{\prime \prime}\right) \cup\left(N \backslash C^{\prime \prime}\right) \supseteq$ $\left(C^{\prime} \cap C^{\prime \prime}\right) \cup\left(C^{\prime} \backslash C^{\prime \prime}\right)=C^{\prime} \in \pi^{-1}\{1\}$. Hence, $\underline{A}^{\prime}(q) \in \pi^{-1}\{1\}$ because we have already seen that $\pi^{-1}\{1\}$ is $\supseteq$-closed. Again, since $\pi$ is well-defined, $\underline{A}^{\prime}(q) \in \pi^{-1}\{1\}$ implies $q \in f\left(\underline{A}^{\prime}\right)$. So, $p, q \in f\left(\underline{A}^{\prime}\right)$, whence $p \wedge q \in f\left(\underline{A}^{\prime}\right)$

\footnotetext{
${ }^{17}$ Such a sentence is also called $\Sigma$-contingent.
} 
because $f\left(\underline{A}^{\prime}\right)$ is deductively closed and $p \wedge q \in X$. It follows that $\pi^{-1}\{1\} \ni$ $\underline{A}^{\prime}(p \wedge q)=C^{\prime} \cap C^{\prime \prime}$.

Proof of Theorem 3, First, suppose that $f$ satisfies (A2), (A3) and (A5) and is consistent and complete. Then Remark 1 teaches that (A4) is satisfied. Also, Lemma 2 already affirms that $\pi^{-1}\{1\}=\mathcal{F}_{f}$. For the first part of the Theorem, it remains to verify that $\pi$ preserves algebraic operations.

$\pi$ preserves meets. Let $C, C^{\prime} \subseteq N$. Since $\pi^{-1}\{1\}$ is both $\supseteq$-closed and $\cap$-closed, we have that

$$
C \cap C^{\prime} \in \pi^{-1}\{1\} \Leftrightarrow\left(C \in \pi^{-1}\{1\}, \quad C^{\prime} \in \pi^{-1}\{1\}\right) .
$$

Because $\pi$ is $\{0,1\}$-valued, we deduce

$$
\pi\left(C \cap C^{\prime}\right)=1 \Leftrightarrow\left(\pi(C)=1, \quad \pi\left(C^{\prime}\right)=1\right) \Leftrightarrow \pi(C) \wedge \pi\left(C^{\prime}\right)=1
$$

and thus

$$
\pi\left(C \cap C^{\prime}\right)=\pi(C) \wedge \pi\left(C^{\prime}\right) .
$$

$\pi$ preserves complements. Let $\underline{A} \in D^{N}$ and $p \in X$. For every $i \in N$, the set $A_{i}$ is consistent and complete, hence

$$
p \in A_{i} \Leftrightarrow \sim p \notin A_{i},
$$

so $\underline{A}(p)=N \backslash \underline{A}(\sim p)=\complement \underline{A}(\sim p)$, or equivalently

$$
\complement \underline{A}(p)=\underline{A}(\sim p) .
$$

On the other hand, $f(\underline{A})$ is consistent and complete, therefore $\sim p \in f(\underline{A})$ if and only if $p \notin f(\underline{A})$. Hence, we finally obtain

$$
\begin{aligned}
& \pi(\complement \underline{A}(p))=1 \Leftrightarrow \pi(\underline{A}(\sim p))=1 \Leftrightarrow \sim p \in f(\underline{A}) \Leftrightarrow p \notin f(\underline{A}) \Leftrightarrow \pi(\underline{A}(p))=0, \\
& \pi(\complement \underline{A}(p))=0 \Leftrightarrow \pi(\complement \underline{A}(p)) \neq 1 \Leftrightarrow \pi(\underline{A}(p)) \neq 0 \Leftrightarrow \pi(\underline{A}(p))=1 .
\end{aligned}
$$

$\pi$ preserves joins. Let $C, C^{\prime} \subseteq N$. First, suppose $\pi(C) \vee \pi\left(C^{\prime}\right)=1$. Then either $\pi(C)=1$ or $\pi\left(C^{\prime}\right)=1$, hence either $C \in \pi^{-1}\{1\}$ or $C^{\prime} \in \pi^{-1}\{1\}$. Therefore, $C \cup C^{\prime}$ will be the superset of an element of $\pi^{-1}\{1\}$, hence by 卫-closedness of $\pi^{-1}\{1\}$, we obtain $C \cup C^{\prime} \in \pi^{-1}\{1\}$, that is $\pi\left(C \cup C^{\prime}\right)=1$.

Next, suppose $\pi(C) \vee \pi\left(C^{\prime}\right)=0$, hence $\pi(C)=\pi\left(C^{\prime}\right)=0$. We have already shown that $\pi$ preserves complements, therefore we deduce that $\pi(\complement C)=\pi\left(\complement C^{\prime}\right)=1$. Since we have also already seen that $\pi$ preserves meets, we obtain that $\pi\left(\complement C \cap \complement C^{\prime}\right)=1 \wedge 1=1$. Using de Morgan's law, $\pi\left(\complement\left(C \cup C^{\prime}\right)\right)=1$, hence, again exploiting that $\pi$ preserves complements, we arrive at $\pi\left(C \cup C^{\prime}\right)=0$.

Hence, $\pi$ is a homomorphism and the first part of the Theorem established.

For the converse part of the Theorem, suppose $\rho: \mathcal{P}(N) \rightarrow 2$ is a homomorphism. We have to verify that $f$ satisfies axioms (A2-A5). (A2) is satisfied by assumption. By definition, $f$ satisfies (A3) and (A5).

To prove (A4), note that $\rho(\varnothing)=0$ and $\rho(N)=1$ since $\rho$ is a homomorphism 18 and by Remark 9 , we can find $\underline{A}, \underline{A}^{\prime} \in D^{N}$ and $p, q \in X$

\footnotetext{
${ }^{18}$ For, since $\rho$ preserves algebraic operations, one has (for an arbitrary $C \subseteq N$ ) $\rho(\varnothing)=\rho(C \cap \complement C)=\rho(C) \wedge \rho(C)^{*}=0$

and

$$
\rho(N)=\rho(C \cup C C)=\rho(C) \vee \rho(C)^{*}=1
$$
}


such that $\underline{A}(p)=\varnothing$ and $\underline{A}^{\prime}(q)=N$. Then, by construction of $f$, both $q \in f\left(\underline{A}^{\prime}\right)$ and $p \notin f(\underline{A})$, so $f\left(\underline{A}^{\prime}\right) \neq \varnothing$ and $f(\underline{A}) \neq X$.

Finally, for every $\underline{A} \in D^{N}$ and $p \in X$, note that $\underline{A}(\sim p)=\complement \underline{\complement} \underline{A}(p)$ by Equation (1), and therefore, using that $\rho$ is a homomorphism,

$$
\begin{aligned}
p \in f(\underline{A}) & \Leftrightarrow \rho(\underline{A}(p))=1 \Leftrightarrow \rho(\complement \underline{A}(p))=0 \Leftrightarrow \rho(\underline{A}(\sim p))=0 \\
& \Leftrightarrow \rho(\underline{A}(\sim p)) \neq 1 \Leftrightarrow \sim p \notin f(\underline{A}) .
\end{aligned}
$$

Hence, $f(\underline{A})$ is complete and algebraically consistent for every $\underline{A} \in D^{N}$.

Proof of Corollary 4. Every shell of a 2-valued homomorphism is an ultrafilter ${ }^{19}$ Therefore, (A2-A5) implies via Theorem 3 that $\mathcal{F}_{f}=\pi^{-1}\{1\}$ is an ultrafilter on $N$.

However, every ultrafilter $\mathcal{F}$ on a finite set $N$ is principal ${ }^{20}$ Hence, if even (A1-A5) are satisfied, then there must be some $i_{f} \in N$ such that $\pi^{-1}\{1\}=\mathcal{F}_{f}=\left\{C \subseteq N: i_{f} \in C\right\}$, hence

$$
p \in f(\underline{A}) \Leftrightarrow \pi(\underline{A}(p))=1 \Leftrightarrow \underline{A}(p) \in \mathcal{F}_{f} \Leftrightarrow i_{f} \in \underline{A}(p) \Leftrightarrow p \in A_{i_{f}}
$$

for all $\underline{A} \in \mathfrak{D}_{f}$ and $p \in X$.

Proof of Lemma 5, Suppose $f$ satisfies (A2-A5). Then $\mathcal{F}_{f}=\pi^{-1}\{1\}$ is non-empty by (A4) and $\cap$-closed by Lemma 2. Therefore, $\sim_{f}$ must be a congruence relation ${ }^{21}$ For all $C \subseteq N$, denote by $|C|$ the equivalence class

by the definition of the complement of a Boolean algebra element.

${ }^{19}$ Cf. e.g. Bell and Slomson 2 , Chapter 1, Lemma 4.7, Theorem 4.9] The proof can be sketched as follows: Every homomorphism $h: \mathcal{P}(N) \rightarrow B$ translates $\cap$ into $\wedge$ and translates $\subseteq$ into $\leq$. (For, if $C \subseteq C^{\prime}$ then $C \cap C^{\prime}=C$, hence $h(C) \wedge h\left(C^{\prime}\right)=h\left(C \cap C^{\prime}\right)=h(C)$, therefore $h(C) \leq h\left(C^{\prime}\right)$.) It is therefore clear that the shell $\mathcal{H}$ of $h$ is $\cap$-closed and $\supseteq$ closed. Moreover, as shown in Footnote 18 on page 10 both $h(\varnothing)=0_{B}$ and $h(N)=1_{B}$, hence $\mathcal{H} \neq \mathcal{P}(N)$ and $\mathcal{H} \neq \varnothing$. Hence, $\mathcal{H}$ is a filter. If, in addition, $h$ is 2 -valued, then

$$
\begin{aligned}
C \in \mathcal{H} & \Leftrightarrow h(C)=1 \Leftrightarrow h(C)^{*}=0 \Leftrightarrow h(\complement C)=0 \\
& \Leftrightarrow h(\complement C) \neq 1 \Leftrightarrow \complement C \notin \mathcal{H},
\end{aligned}
$$

so $\mathcal{H}$ is even an ultrafilter.

${ }^{20}$ Cf. e.g. Bell and Slomson 2 Example 1.3.2 and Exercise 1.3.3] An ultrafilter $\mathcal{F}$ is called principal if and only if there exists some $i \in N$ such that $\mathcal{F}=\{C \subseteq N: i \in C\}$. If $N$ is finite and some ultrafilter $\mathcal{F}$ on $N$ were not principal, then $\{i\} \notin \mathcal{F}$ and hence $N \backslash\{i\}$ for all $i \in N$. Since filters are closed under finite intersections, we get $\bigcap_{i \in N} N \backslash\{i\} \in \mathcal{F}$, albeit $\bigcap_{i \in N} N \backslash\{i\}=N \backslash \bigcap_{i \in N}\{i\}=\varnothing$ by de Morgan's law, a contradiction.

${ }^{21}$ Cf. e.g. Bell and Slomson [2, Chapter 1, proof of Lemma 4.3, proof of Lemma 4.4]. The proof can be summarized as follows: Since $\mathcal{F}_{f}$ is non-empty, $\sim_{f}$ is reflexive. By definition, $\sim_{f}$ is symmetric. Since $\mathcal{F}_{f}$ is $\cap$-closed, $\sim_{f}$ is transitive. (For all $C, C^{\prime}, C^{\prime \prime} \subseteq N$, if there exist $U, V \in \mathcal{F}_{f}$ such that $C \cap U=C^{\prime} \cap U$ and $C^{\prime} \cap V=C^{\prime \prime} \cap V$, then $C \cap U \cap V=$ $C^{\prime \prime} \cap U \cap V$ whilst $U \cap V \in \mathcal{F}_{f}$.) Similarly, since $\mathcal{F}_{f}$ is $\cap$-closed, $\sim_{f}$ respects $\cap$ and $\cup$ : For all $C_{0}, C_{1}, C_{0}^{\prime}, C_{1}^{\prime} \subseteq N$, if there exist $U_{0}, U_{1} \in \mathcal{F}_{f}$ such that $C_{0} \cap U_{0}=C_{0}^{\prime} \cap U_{0}$ and $C_{1} \cap U_{1}=C_{1}^{\prime} \cap U_{1}$, then, by commutativity of $\cap$,

$$
C_{0} \cap C_{1} \cap \underbrace{U_{0} \cap U_{1}}_{\in \mathcal{F}_{f}}=C_{0}^{\prime} \cap C_{1}^{\prime} \cap U_{0} \cap U_{1}
$$

and by distributivity of $\cap, \cup$ also

$$
\left(C_{0} \cup C_{1}\right) \cap \underbrace{U_{0} \cap U_{1}}_{\in \mathcal{F}_{f}}=\left(C_{0}^{\prime} \cup C_{1}^{\prime}\right) \cap U_{0} \cap U_{1} .
$$


of $C$ with respect to $\sim_{f}$. Since $\sim_{f}$ is a congruence relation, the operations $\wedge, \vee,{ }^{*}$, introduced representative-wise via

$$
|C| \wedge\left|C^{\prime}\right|:=\left|C \cap C^{\prime}\right|, \quad|C| \vee\left|C^{\prime}\right|:=\left|C \cup C^{\prime}\right|, \quad|C|^{*}:=|\complement C C|
$$

for all $C, C^{\prime} \subseteq N$, are well-defined. If we define, in addition,

$$
0_{\sim_{f}}:=|\varnothing|, \quad 1_{\sim_{f}}:=|N|
$$

then through straightforward calculations one can check that $\left\langle\mathcal{P}(N) / \sim_{f}, \wedge, \vee,{ }^{*}, 0_{\sim_{f}}, 1_{\sim_{f}}\right\rangle$ is indeed a Boolean algebra.

Proof of Theorem 6, With the notation of the proof of Lemma 5 $\sigma: C \mapsto|C|$ trivially preserves the Boolean operations.

Note that for every $C \subseteq N$, one has

$$
\begin{aligned}
& C \sim_{f} N \Leftrightarrow \exists U \in \mathcal{F}_{f} \quad(C \cap U=N \cap U) \Leftrightarrow \exists U \in \mathcal{F}_{f} \quad(C \cap U=U) \\
\Leftrightarrow & \exists U \in \mathcal{F}_{f} \quad U \subseteq C,
\end{aligned}
$$

hence, due to the $\supseteq$-closedness of $\mathcal{F}_{f}$, we obtain

$$
|C|=|N| \Leftrightarrow C \sim_{f} N \Leftrightarrow C \in \mathcal{F}_{f} .
$$

Therefore $\sigma^{-1}\left\{1_{\sim f}\right\}=\sigma^{-1}\{|N|\}=\mathcal{F}_{f}$.

For the converse part of the Theorem, the same argument as in the proof of Theorem 3 shows that $f$ satisfies axioms (A2-A5).

Finally, suppose $f$ were not algebraically consistent. Then there would be some $\underline{A} \in D^{N}$ and $p \in X$ such that both $\sim p \in f(\underline{A})$ and $p \in f(\underline{A})$. Hence $\tau(\underline{A}(p))=f(\underline{A})(p)=1$ as well as $\tau(\underline{A}(\sim p))=g(\underline{A})(\sim p)=1$. However $\underline{A}(\sim p)=\complement \underline{A}(p)$ by Equation (1), so $\tau(\complement \underline{\complement} \underline{A}(p))=1$. On the other hand, since $\tau$ is a homomorphism and $\tau(\underline{A}(p))=1$, one has $\tau(\complement \underline{A}(p))=0$, contradiction. This proves the algebraic consistency of $f$.

Proof of Corollary 7, As the shell of a homomorphism, $\mathcal{F}_{f}$ is a filter.22

For every filter $\mathcal{F}$ on a finite set $N$, there exists some $M \subseteq N$ such that $\mathcal{F}=\{C \subseteq N: M \subseteq C\}{ }^{23}$ Hence, if (A1-A5) are satisfied, then there

Finally, for all $C, C^{\prime} \subseteq N$, if there exists $U \in \mathcal{F}_{f}$ such that $C \cap U=C^{\prime} \cap U$, then

$$
U=(U \cap C) \cup(U \cap \complement C)=\left(U \cap C^{\prime}\right) \cup(U \cap \complement C),
$$

whence on the one hand

$\complement C^{\prime} \cap U=\complement C^{\prime} \cap\left(\left(U \cap C^{\prime}\right) \cup(U \cap \complement C)\right)=\underbrace{\left(\complement C^{\prime} \cap U \cap C^{\prime}\right)}_{=\varnothing} \cup\left(\complement C^{\prime} \cap U \cap \complement C\right)=\complement C^{\prime} \cap \complement C \cap U$,

and symmetrically (by interchanging the roles of $C$ and $C^{\prime}$ ), one obtains on the other hand

$$
\complement C \cap U=\complement C \cap \complement C^{\prime} \cap U=\complement C^{\prime} \cap \complement C \cap U,
$$

hence $\complement C^{\prime} \cap U=\complement C \cap U$.

${ }^{22}$ Cf. e.g. Bell and Slomson [2, Chapter 1, Lemma 4.7]; see Footnote 19 on page 11

${ }^{23}$ Filters are closed under finite intersections, hence $\bigcap \mathcal{F} \in \mathcal{F}$ for every filter on a finite set $N$. This implies, since filters are closed under supersets, $\{C \subseteq N: \bigcap \mathcal{F} \subseteq C\} \subseteq \mathcal{F}$. Trivially, the converse inclusion also holds. Hence $\mathcal{F}=\{C \subseteq N: \cap \mathcal{F} \subseteq C\}$ for every filter $\mathcal{F}$ on a finite set $N$. 
must be some $M_{f} \subseteq N$ such that $\pi^{-1}\{1\}=\mathcal{F}_{f}=\left\{C \subseteq N: M_{f} \subseteq C\right\}=$ $\bigcap_{i \in M_{f}}\{C \subseteq N: i \in C\}$, so

$p \in f(\underline{A}) \Leftrightarrow \pi(\underline{A}(p))=1 \Leftrightarrow \underline{A}(p) \in \mathcal{F}_{f} \Leftrightarrow(\forall i \in M_{f} \underbrace{i \in \underline{A}(p)}_{\Leftrightarrow p \in A_{i}}) \Leftrightarrow p \in \bigcap_{i \in M_{f}} A_{i}$

for all $\underline{A} \in D^{N}$ and $p \in X$.

\section{Conclusion}

Under the assumptions of agenda richness, universality and systematicity, we have established a correspondence between deductively closed judgment aggregators and Boolean algebra homomorphisms on the power-set of the electorate. Moreover, we have shown that complete judgment aggregators correspond to 2-valued Boolean algebra homomorphisms on the power-set of the electorate.

As is well-known, Boolean algebra homomorphisms can be partially characterized through their shells, and (2-valued) homomorphism shells are the same as (ultra)filters. We have shown that the shell of a homomorphism induced by a judgment aggregator is just the set of all winning coalitions. Hence the set of winning coalitions is always a filter - and even an ultrafilter if the judgment aggregator is complete. This provides an explanation for the effectiveness of the (ultra)filter method in social choice theory.

From the (ultra)filter property of the set of winning coalitions, one can easily derive impossibility theorems for judgment aggregators on finite electorates, even without requiring preservation of unanimity (Pareto principle): Assuming agenda richness, universality and systematicity, the oligarchies are the only non-trivial aggregators, and the dictatorships are the only complete aggregators.

\section{References}

[1] F. Aleskerov. Arrovian aggregation models, volume 39 of Theory and Decision Library. Series B: Mathematical and Statistical Methods. Kluwer Academic Publishers, Boston, MA, 1999.

[2] J.L. Bell and A.B. Slomson. Models and ultraproducts. An introduction. NorthHolland, Amsterdam, 1969.

[3] D.J. Brown. Aggregation of preferences. Quarterly Journal of Economics, 89(3):456469, August 1975.

[4] F. Dietrich and C. List. Judgment aggregation without full rationality. Social Choice and Welfare, 31(1):15-39, 2008.

[5] F. Dietrich and Ph. Mongin. The premiss-based approach to logical aggregation. Les Cahiers de Recherche 886, HEC Paris, December 2007.

[6] E. Dokow and R. Holzman. Aggregation of binary evaluations. Journal of Economic Theory, 145(2):495-511, 2010.

[7] D. Eckert and C. Klamler. A simple ultrafilter proof for an impossibility theorem in judgment aggregation. Economics Bulletin, 29(1):320-328, 2009.

[8] P.C. Fishburn. Arrow's impossibility theorem: concise proof and infinite voters. Journal of Economic Theory, 2(1):103-106, 1970.

[9] G.T. Guilbaud. Les théories de l'intérêt général et le problème logique de l'agrégation. Economie Appliquée, 5(4):501-584, 1952.

[10] G.T. Guilbaud. Theories of the general interest and the logical problem of aggregation. Journal Electronique d'Histoire des Probabilités et de la Statistique, 4(1), 2008. 
[11] B. Hansson. The existence of group preference functions. Public Choice, 38:89-98, 1976.

[12] K.H. Kim and F.W. Roush. Introduction to mathematical consensus theory, volume 59 of Lecture Notes in Pure and Applied Mathematics. Marcel Dekker, New York, 1980.

[13] A.P. Kirman and D. Sondermann. Arrow's theorem, many agents, and invisible dictators. Journal of Economic Theory, 5(2):267-277, 1972.

[14] L. Lauwers and L. Van Liedekerke. Ultraproducts and aggregation. Journal of Mathematical Economics, 24(3):217-237, 1995.

[15] C. List and C. Puppe. Judgment aggregation: A survey. In P. Anand, C. Puppe, and P.K. Pattanaik, editors, Oxford Handbook of Rational and Social Choice. Oxford University Press, Oxford, 2009.

[16] B. Monjardet. On the use of ultrafilters in social choice theory. In P.K. Pattanaik and M. Salles, editors, Social Choice and Welfare (Caen, 1980), Contributions to Economic Analysis. 145, pages 73-78. North-Holland, Amsterdam, 1983.

[17] K. Nehring and C. Puppe. The structure of strategy-proof social choice. Part II: Non-Dictatorship, Anonymity and Neutrality. Working Paper, Institut für Wirtschaftstheorie und Operations Research, Universität Karlsruhe, 2005. 